\title{
Effects of dimethyl carbonate-induced autophagic activation on follicular development in the mouse ovary
}

\author{
ZONGHAO TANG $^{1}$, ZHENGHONG ZHANG $^{1}$, YEDONG TANG ${ }^{1}$, \\ LINGBIN QI $^{1}$, FAFU YANG ${ }^{2}$ and ZHENGCHAO WANG ${ }^{1}$ \\ ${ }^{1}$ Provincial Key Laboratory for Developmental Biology and Neurosciences, \\ College of Life Sciences, Fujian Normal University; ${ }^{2}$ College of Chemistry and \\ Chemical Engineering, Fujian Normal University, Fuzhou, Fujian 350007, P.R. China
}

Received March 8, 2017; Accepted August 24, 2017

DOI: $10.3892 / \mathrm{etm} .2017 .5328$

\begin{abstract}
Dimethyl carbonate (DMC) is a widely used industrial chemical, which may be increasingly used in the future. However, its toxicity profile remains largely unknown. The present study was designed to investigate the effects of DMC exposure on the ovaries and the effect of autophagy activation on follicular development. Rats were randomly divided into a control group and low, medium and high dose DMC groups (all $n=10)$. Histological analyses identified no marked differences in the rate of apoptosis between the control and low dose groups; however, marked apoptosis occurred in the medium and high dose groups. The expression of cleaved caspase-3 was significantly increased in the medium and high dose groups, which was consistent with changes observed in the expression of Bcl-2 and Bax. These results indicated that DMC exposure induces toxicity on ovarian function via the induction of apoptosis. The increased expression of the autophagy-related proteins light chain 3II, beclin-1 and p62 following exposure to DMC further indicated that autophagy was activated primarily in the granulosa cells of ovarian follicles in a dose-dependent manner. In addition, the changes in the expression of hypoxia inducible factor $1 \alpha$ subunit (HIF-1 $\alpha$ ) and its target protein BCL2 interacting protein 3 (BNIP3) indicated that they may serve a role in the follicular development process induced by DMC. The results of the current study demonstrated that DMC exposure activated autophagy in the ovarian tissue. Furthermore, exposure to low doses of DMC may protect follicular development by activating the HIF-1 $\alpha /$ BNIP3 signaling pathway. Taken together, these results indicate that exposure to medium and high doses of DMC induced follicular
\end{abstract}

Correspondence to: Dr Zhengchao Wang, Provincial Key Laboratory for Developmental Biology and Neurosciences, College of Life Sciences, Fujian Normal University, 8 Shangsan Road, Fuzhou, Fujian 350007, P.R. China

E-mail: zcwang@fjnu.edu.cn

Key words: dimethyl carbonate, autophagy, apoptosis, follicular development, ovary atresia by activating the apoptotic signaling pathway. This may be an important mechanism of regulating follicular development and ovarian function in mammals.

\section{Introduction}

Dimethyl carbonate (DMC) is an environmentally friendly organic solvent, which may have a wide range of potential applications, such as being used as a new solvent or fuel additive (1). Due to high market demand and environmental regulations, DMC may be a potential substitute for the organic solvents and chemical midbodies that are currently used, for example DMC could be used as a substitute of methyl chloroform for organic solvents or carbonyl chloride for the production of polycarbonate and carbamic acid ester. However, compared with other commercially used chemicals, the toxicity of DMC remains unknown. It has been demonstrated that 10 day inhalation exposure to 3,000 ppm DMC in pregnant female mice during days $6-15$ of pregnancy for $6 \mathrm{~h}$ /day reduces the weight of mothers and fetuses, and increases the number of malformed and hypogenetic fetuses (2). In recent years, the adverse health effects of various chemicals, including the toxicity of chemicals to the female reproductive system, have become more topical. It has been demonstrated that various industrially used chemicals may disrupt hormonal regulation and follicular development in the mammalian ovary (3). Environmental contaminants have been detected in the ovarian follicular fluid, further indicating the effect of such chemicals on ovarian function (4). However, the toxicity of DMC on the reproductive system remains largely unknown.

Autophagy is a primary cytoprotective mechanism and is characterized by the formation of isolation membranes. These membranes sequester unnecessary cellular components or damaged organelles, including mitochondria and endoplasmic reticulum, and form autophagosomes to recycle building blocks for cell survival when the cell is under stress $(5,6)$. Previous studies have demonstrated that autophagy serves a role in regulating testicular homeostasis following exposure to toxins $(7,8)$; however few relevant studies have investigated the effects of toxins on the ovaries. In the testes, autophagy is generally induced by the upregulation of reactive oxygen species (ROS) in tissues following exposure to toxins (9). 
Previous studies have also revealed that exposure to toxins is associated with ROS accumulation in the ovaries $(10,11)$. In addition, it has been demonstrated that the hypoxia inducible factor $1 \alpha$ subunit $(\mathrm{HIF}-1 \alpha)$ signaling pathway serves a role in regulating cellular ROS levels by stimulating mitochondrial selective autophagy to reduce ROS production $(12,13)$. Other studies have demonstrated that HIF-1 $\alpha$ activation is a self-protective mechanism that occurs under adverse conditions, as it upregulates genes encoding glucose transporters and glycolytic enzymes, downregulates mitochondrial genes (14) and activates autophagic signaling pathways (15). In mammalian ovaries, HIF-1 $\alpha$ is ubiquitously expressed during various stages of folliclular and corpus luteum development, and serves a pivotal role in the maintenance of ovarian homeostasis $(16,17)$.

Due to the important role served by HIF-1 $\alpha$ signaling in ovarian homeostasis, it was hypothesized that the HIF-1 $\alpha$ signaling pathway serves a role in the regulation of ovarian function following exposure to DMC. Therefore, the present study investigated the histological changes and expression pattern of apoptosis-related proteins in the ovary to determine whether DMC exposure induces injury in the ovary and assessed the role served by the HIF-1 $\alpha$ signaling pathway in doing this.

\section{Materials and methods}

Animals and treatment. A total of 40 healthy adult female Kunming mice weighing 18-22 g (age, 6-8 weeks) were purchased from Wushi Experimental Animal Supply Co. Ltd. (Fuzhou, China). Mice were kept in a room with a constant temperature of $21-25^{\circ} \mathrm{C}$, a relative humidity of $40-60 \%$ and a $12 \mathrm{~h}$ light/dark cycle, and fed a standard diet and tap water ad libitum. Mice were housed in cages and each cage contained 3-4 mice. Prior to grouping, the toxicity of DMC was pre-tested by extra experiments (results not shown). According to the results of the pre-test, all mice were randomly distributed into the following 4 groups (all $n=10)$ : A control group (Ctrl) treated with corn oil, a low dose treatment group (group A) treated with $0.06 \mathrm{~g} / \mathrm{kg} \mathrm{DMC}$; a medium dose treatment group (group B) treated with $0.6 \mathrm{~g} / \mathrm{kg} \mathrm{DMC}$; and a high dose treatment group (group C) treated with $6.0 \mathrm{~g} / \mathrm{kg}$ DMC. All mice in the treatment groups were administered DMC (Sinopharm Chemical Reagent Co., Ltd, Shanghai, China) via gavage. DMC is unable to dissolve in water, therefore corn oil (Shandong Luhua Group Co., Ltd., Laiyang, China) was used as a solvent and different dilutions were prepared prior to administration. The dilutions were $0.3,3$ and $30 \%(\mathrm{v} / \mathrm{v})$ and the volume of treatment dosages was $0.4 \mathrm{ml}$. All mice were administrated an equal volume of solution by gavage every 2 days over a treatment period of 30 days. The mice in the control group were treated with corn oil alone via gavage. The current study was approved by the Animal Ethical and Welfare Committee of Funjian Normal University (Fujian, China).

Tissue preparation and histopathological examination. At the end of treatment, mice were sacrificed by cervical dislocation. The bilateral ovaries were immediately removed and weighed. The left ovary of each animal was used for histopathological examination and the right ovary was used to examine the expression of different functional proteins. Ovarian tissues for histological study were fixed in $4 \%$ paraformaldehyde at $37^{\circ} \mathrm{C}$ for $24 \mathrm{~h}$, then dehydrated and embedded in paraffin; sections $5-\mu \mathrm{m}$ thick were subsequently prepared. Ovary sections were dewaxed and rehydrated prior to staining with hematoxylin. The sections were stained with hematoxylin at room temperature for 20-30 sec and then observed under a light microscope.

Immunohistochemistry of light chain (LC)-3 and HIF-1 $\alpha$. Immunohistochemical staining of LC-3 and HIF-1 $\alpha$ were performed following the manufacturer's protocols and previously reported studies $(18,19)$. Briefly, paraffin-embedded tissue sections (prepared as described above) were dewaxed and rehydrated. Sections were then subjected to antigen microwave antigen retrieval using $0.01 \mathrm{M}$ citric acid buffer at $100^{\circ} \mathrm{C}$ for $10 \mathrm{~min}$. Endogenous peroxide was inhibited by incubation of the sections in $3 \% \mathrm{H}_{2} \mathrm{O}_{2}$ for $30 \mathrm{~min}$. Sections were then incubated in $5 \%$ bovine serum albumin at room temperature for $\sim 20 \mathrm{~min}$ to block non-specific reactions. Primary antibodies $(50 \mu \mathrm{l})$ were incubated overnight at $4^{\circ} \mathrm{C}$. The primary antibodies used were anti-LC-3B antibody (1:500, cat. no. ab48394, Abcam, Cambridge, MA, USA) and anti-HIF-1 $\alpha$ antibody (1:400, cat. no. sc-53546, Santa Cruz Biotechnology Inc., Dallas, TX, USA). Following washing with PBS, slides were incubated with the secondary antibodies (PV-9001 and PV-9002, OriGene Technologies, Beijing, China) at room temperature for $20 \mathrm{~min}$, respectively. For visualization, all sections were stained with diaminobenzidine tetrahydrochloride chromogen at room temperature for 3-6 min. All sections were then counterstained with hematoxylin at room temperature for $5 \mathrm{~min}$, dehydrated and mounted. The sections were observed under a light microscope.

Western blot analysis of cleaved caspase-3, Bcl-2, Bax, LC-3II, beclin-1, HIF-1 $\alpha$ and BNIP3 expression. Ovarian tissues from each group were homogenized in ice-cold radioimmunoprecipitation assay lysate buffer (cat. no. P0013, Beyotime Institute of Biotechnology, Haimen, China) and centrifuged at $15,000 \mathrm{x} \mathrm{g}$ for $15 \mathrm{~min}$ at $4^{\circ} \mathrm{C}$. The supernatant was then collected. Nuclear proteins were extracted using a kit from Beyotime Institute of Biotechnology (Nuclear and Cytoplasmic Protein Extraction kit, cat. no. P0027) and protein concentrations were determined using a BCA Protein assay kit (cat. no. P0011, Beyotime Institute of Biotechnology). Protein samples were diluted into equal concentrations and $20 \mu \mathrm{g}$ protein samples were then subjected to $12 \%$ SDS-PAGE gel electrophoresis and electrophoretically transferred onto polyvinylidene difluoride membranes (Pall Life Sciences, Port Washington, NY, USA). Membranes were washed with TBS with 0.2\% Tween-20 (TBST; Sigma-Aldrich; Merck KGaA, Darmstadt, Germany) and blocked with 5\% nonfat milk in Tris buffered saline-Tween-20 (TBST, pH 7.4) for $1 \mathrm{~h}$ at room temperature. Membranes were then incubated with anti-cleaved caspase-3 antibody (1:1,000; cat. no. 5A1E; Cell Signaling Technology, Danvers, MA, USA), anti-Bcl-2 antibody (1:1,000; cat. no. 12789; Proteintech Group, Wuhan, China), anti-Bax antibody (1:2,000; cat. no. 60,267; Proteintech Group), anti-LC-3II antibody (1:1,000; cat. no. ab48394; Abcam), anti-Beclin-1 antibody (1:1,500; cat. no. 11306; Proteintech Group), anti-p62 antibody (1:1,000, cat. no. ab56416; Abcam), anti-HIF-1 $\alpha$ antibody (1:500, cat. 
no. sc-53,546; Santa Cruz Biotechnology), anti-BNIP3 antibody $(1: 1,000$, cat. no. ab10433; Abcam) and anti- $\beta$-actin antibody (1:4,000; cat. no. 66,009; Proteintech Group) overnight at $4^{\circ} \mathrm{C}$. Following three washes with TBST, the membrane was incubated in horseradish peroxidase-conjugated goat anti-rabbit (cat. no. A0208) or mouse (cat. no. A0216) immunoglobulin (1:4,000, Beyotime Institute of Biotechnology) for $1 \mathrm{~h}$ at room temperature. Bands were then visualized using an enhanced chemiluminscence kit (Beyotime Institute of Biotechnology). Blots were quantified using Image J 1.49 software (National Institutes of Health, Bethesda, MD, USA).

Statistical analysis. Data are presented as the mean \pm standard error of the mean. The significance of differences of mean values within and between multiple groups was evaluated using one-way analysis of variance, followed by Tukey's range test. $\mathrm{P}<0.05$ was determined to indicate a statistically significant difference. ${ }^{\#} \mathrm{P}<0.05$ vs. the control, ${ }^{\&} \mathrm{P}<0.05$ vs. Group $\mathrm{A}$, ${ }^{*} \mathrm{P}<0.05$ vs. Group B.

\section{Results}

Effect of DMC exposure on ovarian histopathology. To clarify whether the exposure of DMC led to impairments in the ovary, the morphology of ovaries from the different groups of mice was examined (Fig. 1). Staining indicated that the granulosa cells of developing follicles from mice in the control group were well organized and tightly arranged around the inner of the follicular theca and that the connection between granulosa cells and oocytes was well maintained (Fig. 1A and B). The morphological structure of the ovaries was largely maintained in group A (Fig. 1C and D); however, it was notably altered in groups B (Fig. 1E and F) and C (Fig. 1G and H) compared with the control group. The primary histopathological changes following exposure to DMC included follicular atresia and dispersal of granulosa cells in the developing follicles.

DMC exposure increases apoptosis in ovarian tissue. To determine whether DMC exposure activates the apoptotic pathway, the expression of apoptosis-related proteins was measured using western blotting. Bcl-2 primarily functions as a factor to maintain the survival of cells, whereas Bax promotes the activation of capase-3 and induces the apoptosis of cells (20).

The results demonstrated that the expression of cleaved caspase-3, a marker of cellular apoptosis, increased significantly following DMC treatment, in a dose-dependent manner (Fig. 2). Further analysis indicated that the Bcl-2 expression was significantly increased in group A and significantly decreased in groups $\mathrm{B}$ and $\mathrm{C}$ (Fig. 3A and $\mathrm{B}$ ), whereas the expression of Bax was similar to the control group in group A and significantly increased in groups B and C (Fig. 3C). The ratio of Bax to $\mathrm{Bcl}-2$ indicates whether cells are pro-survival or pro-death. It was observed that the ratio of $\mathrm{Bax} / \mathrm{Bcl}-2$ exhibited a similar pattern to that of cleaved caspase-3 (Figs. 2B and 3D). These results suggest that low doses of DMC do not affect cellular apoptosis, whereas higher doses of DMC increase the rate of apoptosis in the mammalian ovaries.

DMC exposure activates autophagy in the ovaries. Previous studies have identified the involvement of autophagy in the
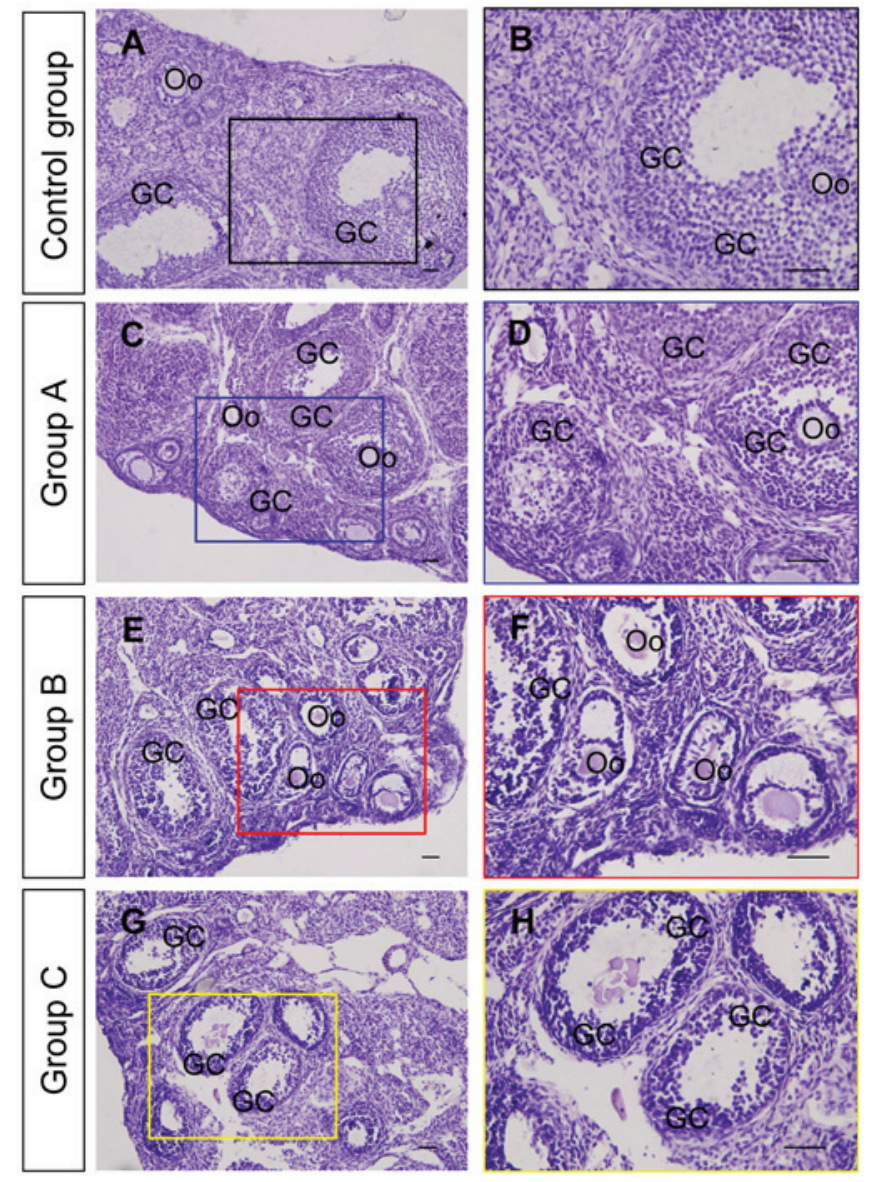

Figure 1. Ovarian histological examination using hematoxylin. (A and B) Ovaries from the control group. (C and D) Ovaries from group A. (E and F) Ovaries from group B ( $\mathrm{G}$ and $\mathrm{H})$. Ovaries from group C. Scale bar $=100 \mu \mathrm{m}$. Coloured boxes on the left side panels indicate the area exhibited on the right side panels. GC, granulosa cell; Oo, oocyte; DMC, dimethyl carbonate; control group, mice treated with corn oil; group A, mice treated with a low dose of DMC; group B, mice treated with a medium dose of DMC; group $\mathrm{C}$, mice treated with a high dose of DMC.

regulation of reproductive system following exposure to toxins $(1,2)$. To determine the possible role of autophagy in the ovaries following DMC exposure, the expression of LC3-II, a marker of autophagy, was determined using immunostaining (Fig. 4) and western blotting (Fig. 5). Immunohistochemistry detected positive staining of LC-3II in groups A, B and C following DMC exposure (Fig. 4) and identified that LC3-II expression was located specifically in the ovarian granulosa cells (Fig. 4). Western blot analysis demonstrated that DMC exposure significantly activated autophagy in the ovaries following DMC treatment in a dose-dependent manner (Fig. 5).

To further determine the involvement of autophagy in ovarian injury following exposure to DMC, the expression of Beclin-1 and p62 were also measured. Beclin-1 expression was significantly increased in group A but was significantly decreased in groups B and C, compared with the control group (Fig. 6A and B), indicating that Beclin-1, as an autophagy inducing protein, is involved in the induction of autophagy. p62 expression was significantly increased following DMC treatment in a dose-dependent manner (Fig. 6A and C). This suggested that p62 is a marker protein involved in the degradation of autophagosomes, the upregulation of which 

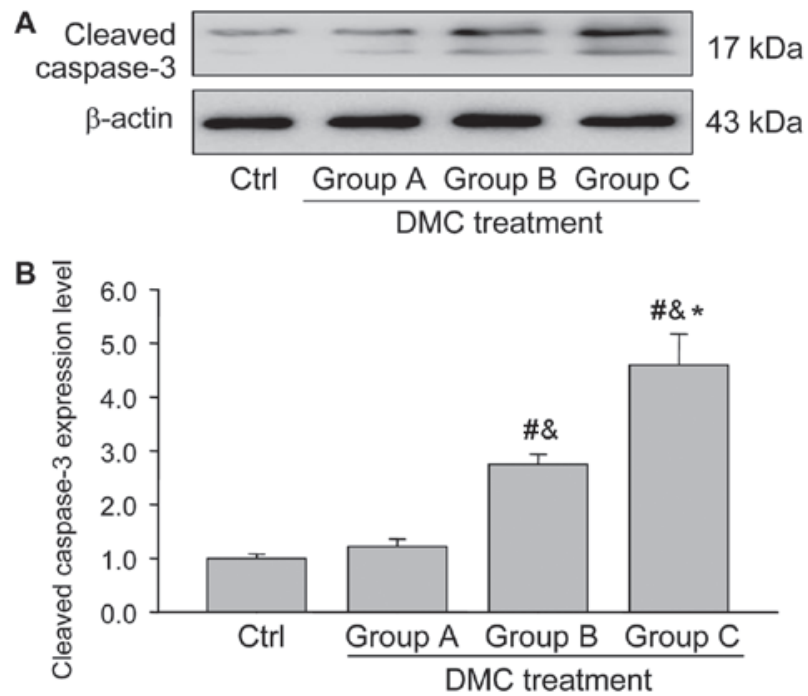

Figure 2. Cleaved caspase-3 expression in the ovaries from each group. (A) Western blots presenting the expression of cleaved caspase-3 in the different groups. (B) Quantification of cleaved caspase-3 protein expression normalized to $\beta$-actin. Data are expressed as the mean \pm standard error of the mean. One-way analysis of variance was used to analyze the data. ${ }^{*} \mathrm{P}<0.05$ vs. control; ${ }^{\circledR} \mathrm{P}<0.05$ vs. Group A; ${ }^{*} \mathrm{P}<0.05$ vs. Group B. Ctrl, control group treated with corn oil; group A, mice treated with a low dose of DMC; group $\mathrm{B}$, mice treated with a medium dose of DMC; group C, mice treated with a high dose of DMC; DMC, dimethyl carbonate.

indicates an accumulation of autophagosomes. In these results, it was observed that p62 was involved in autophagic vesicle accumulation.

HIF-1 $\alpha / B N I P 3$ signaling is involved in DMC-induced autophagy. Previous studies have demonstrated that the HIF-1 $\alpha$ signaling pathway is activated under adverse conditions to maintain cell survival and does so either by activating survival related genes or initiating autophagy $(3,4)$. To determine whether HIF-1 $\alpha$ is involved in the regulating autophagy in the ovary, the expression and localization of HIF-1 $\alpha$ were detected using immunohistochemistry and western blotting. The results indicated that the HIF- $1 \alpha$ staining intensity in granulosa cells from group A was increased compared with those from groups B and C (Fig. 7), which was also consistent with the results of the western blot analysis (Fig. 8A and B).

In addition, the expression of BNIP3, a protein downstream from HIF-1 $\alpha$, was also determined using western blotting and the results demonstrated that its expression was significantly increased in group A compared with the control group and groups B and C (Fig. 8C), which was similar to the expression pattern of total or nuclear HIF-1 $\alpha$ (Fig. 8D and E). These results suggest that $\mathrm{HIF}-1 \alpha / \mathrm{BNIP} 3$ signaling was activated in the ovaries of group A and may be involved in DMC-induced autophagy.

\section{Discussion}

To the best of our knowledge, the present study was the first to investigate the potential toxic effects of DMC exposure on follicular development in the ovary. The results of histological examination indicated that high doses of DMC administered by gavage significantly impaired the development of follicles.
This was indicated primarily by the arrangement of granulosa cells and the irregular shape of oocytes within the ovarian follicles following DMC administration. In addition, the expression of apoptotic related proteins, including cleaved capase-3 and Bax were significantly increased, whereas Bcl-2 was concomitantly decreased. Compelling evidences have been demonstrated that Bax is the activator of caspase- 3 and Bcl-2 is the inhibitor of caspase-3 (20). Thus, these results further demonstrated that high doses of DMC induced ovarian follicular atresia by activating the caspase-3 dependent apoptotic-signaling pathway. At present, the organ-specific toxicity of DMC has not been evaluated, although acute toxicity studies have been conducted $(5,6)$. Indeed, the material safety data sheet for DMC states that its toxicological properties remain untested (6). It has been suggested that chemicals exhibiting low general toxicity may impair the reproductive system of mammals $(7,8)$, which is consistent with the results of the current study.

In female mammals, reproductive capacity is largely dependent on the normal functions of the ovary, including the successful development of follicles (21). Autophagy is a major catabolic pathway that serves a role in the delivery of misfolded proteins and damaged organelles to lysosomes and is frequently induced in response to nutrient starvation or under stressful conditions, including hypoxic stress, heat stress and chemical insults (10-12). The induction of autophagy may adversely affect cellular physiology; its induction may lead to cell survival, however, excessive autophagy or accumulation of autophagosomes may also activate caspase-3 dependent apoptosis (22). During the induction of autophagy, autophagy-related proteins, including Atg5, Atg6, Atg7 and Beclin-1 regulate the process of autophagosome formation, transportation and fusion (23). It has been suggested that the conversion of LC-3I into LC-3II may be a marker of autophagy and that LC-3II expression is associated with the extent of autophagosome formation (13). Previous studies have identified the regulatory role of autophagy in the impairment of the reproductive system of mammals induced by exposure to chemicals (14-17), however, the majority of studies have focused on the effects of such chemicals in the testes. In the present study, to investigate whether autophagy serves a role in regulating ovarian functions following exposure to DMC, LC-3II expression in the ovaries was measured. The results demonstrated that LC-3II expression was significantly upregulated in all treatment groups compared with the control group. This indicates that autophagy is significantly increased following DMC treatment even in groups exposed to low doses of DMC. To determine the primary site of autophagy, LC-3II distribution was determined in the ovaries of all different groups. The results indicated that the staining intensities of ovarian follicles from the DMC treatment groups were stronger than the control group, suggesting that the ovarian follicles may be the primary site of DMC induced-autophagy. The expression of LC-3II was significantly increased in group A and was accompanied by the maintenance of apoptosis, implying that autophagy may serve a cytoprotective role following exposure to low doses of DMC. However, LC-3II expression was also significantly increased in a dose-dependent manner in the medium and high dose treatment groups and the expression of apoptosis-related proteins was significantly increased, as indicated by the increase in the 


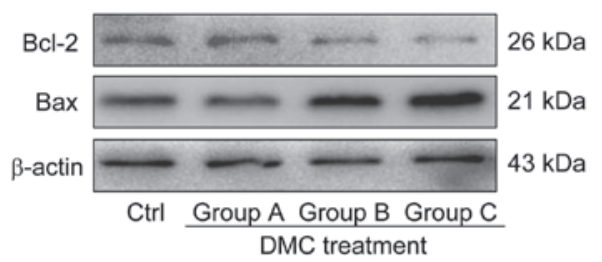

C

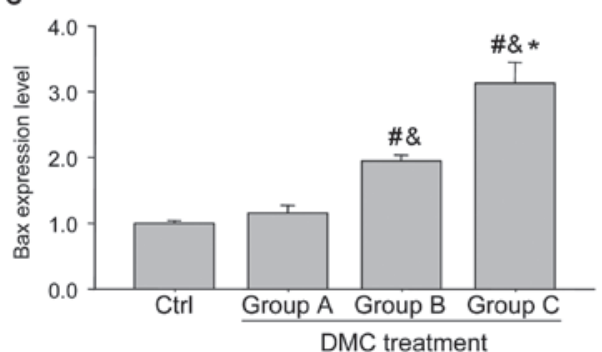

B

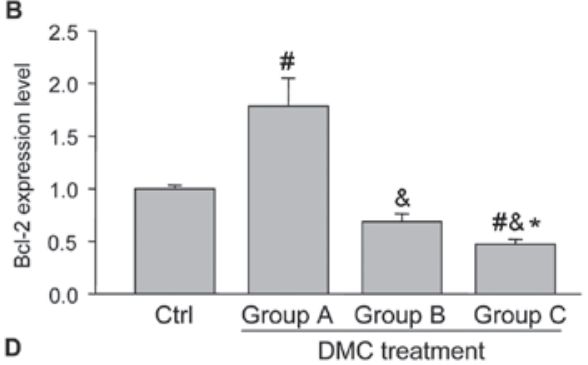

D

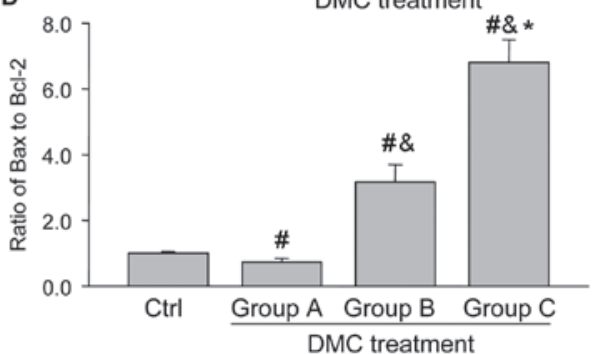

Figure 3. Bcl-2 and Bax expression in the ovaries from each group. (A) Western blotting presenting the expression of Bcl-2 and Bax in the different groups. (B) Quantification of Bcl-2 expression normalized to $\beta$-actin. (C) Quantification of Bax expression normalized to $\beta$-actin. (D) Ratio of Bax to Bcl-2 expression. Data are expressed as the mean \pm standard error of the mean. One-way analysis of variance was used to analyze the data. ${ }^{*} \mathrm{P}<0.05$ vs. control; ${ }^{\mathrm{P}}<0.05$ vs. group A; "P<0.05 vs. group B. Ctrl, control group treated with corn oil; group A, mice treated with a low dose of DMC; group B, mice treated with a medium dose of DMC; group C, mice treated with a high dose of DMC; DMC, dimethyl carbonate.

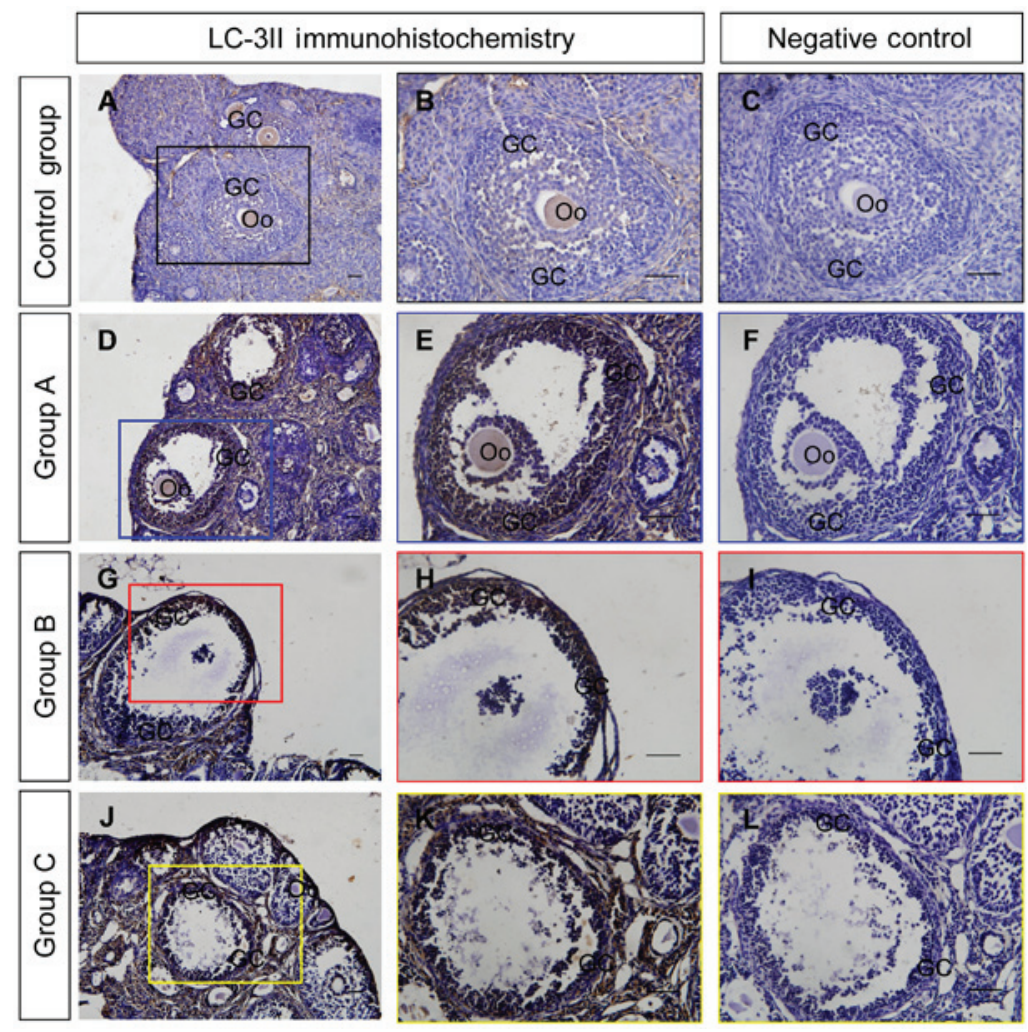

Figure 4. Immunohistochemical examination of LC-3II in the ovaries from each group. LC-3II immunohistochemical signals are brown and the counterstaining background are blue. Negative controls remained unstained, as they lacked primary antibody. (A and B) LC-3II immunohistochemical staining in ovaries from the control group. (C) Negative control of the control group. (D and E) LC-3II immunohistochemical staining in ovaries from group A. (F) Negative control of group A. (G and H) LC-3II immunohistochemical staining in ovaries from group B. (I) Negative control of group B. (J and K) LC-3II immunohistochemistry in ovaries from group C. (L) Negative control of group C. Scale bar $=100 \mu \mathrm{m}$. Coloured boxes on the left side panels indicate the area exhibited on the middle and right side panels. GC, granulosa cell, Oo, oocyte; LC-3II, light chain 3 II; control group, mice treated with corn oil; group A, mice treated with a low dose of DMC; group B, mice treated with a medium dose of DMC; group C, mice treated with a high dose of DMC; DMC, dimethyl carbonate.

Bax/Bcl-2 expression ratio and cleaved caspase-3 expression. Previous studies have demonstrated that exposure to high doses of toxins may disrupt the process of autophagy $(2,7)$. To explore whether autophagy was induced following exposure to DMC, the present study measured the expression of Beclin-1, a protein involved in the initiation of autophagy (24) and 
A
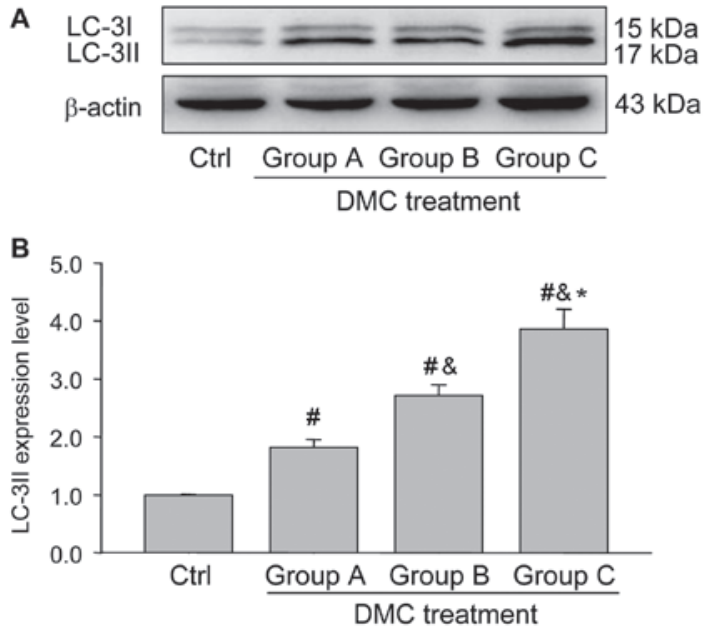

Figure 5. Western blot analysis of LC-3I and LC-3II expression in the ovaries from each group. (A) Western blotting presenting the expression of LC-3I and LC-3II of the different groups. (B) Quantification of LC3II expression normalized to $\beta$-actin. Data are expressed as the mean \pm standard error of the mean. One-way analysis of variance was used to analyze the data. ${ }^{\text {"P }} \mathrm{P}<0.05$ vs. control; ${ }^{\&} \mathrm{P}<0.05$ vs. Group $\mathrm{A} ;{ }^{*} \mathrm{P}<0.05$ vs. Group B. Ctrl, control group treated with corn oil; group A, mice treated with a low dose of DMC; group $\mathrm{B}$, mice treated with a medium dose of DMC; group $\mathrm{C}$, mice treated with a high dose of DMC; DMC, dimethyl carbonate; LC-3, light chain 3.
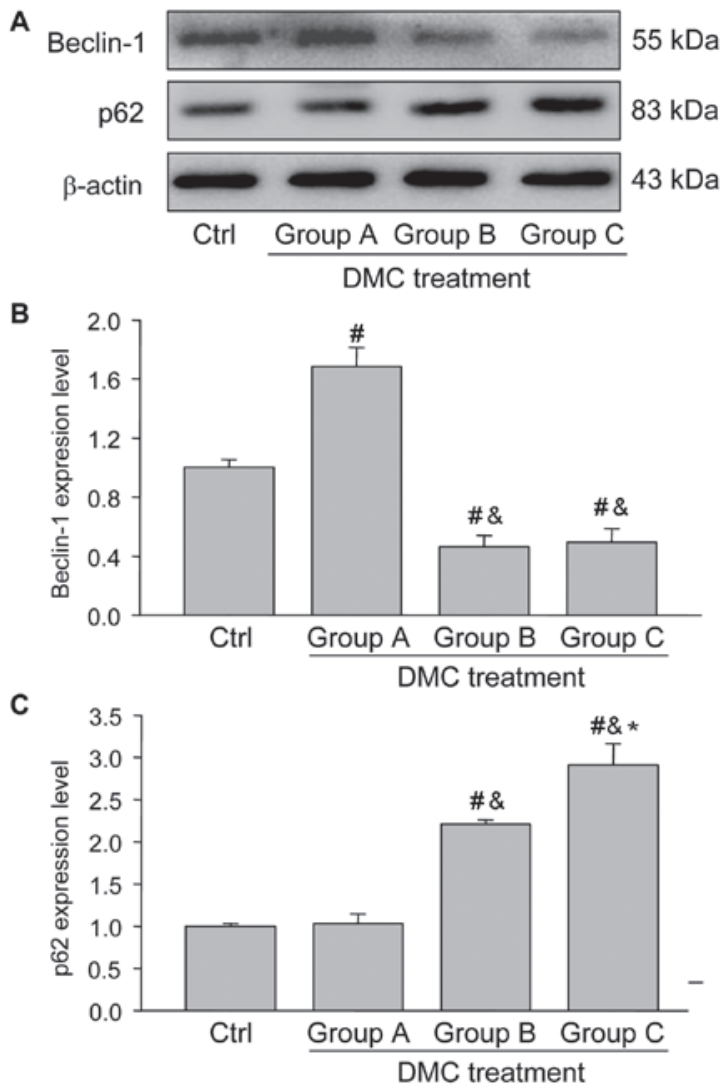

Figure 6. Western blot analysis of Beclin-1 and p62 expression in the ovaries from each group. (A) Western blotting indicating the expression of Beclin-1 and p62 in the different groups. (B) Quantification of Beclin-1 expression normalized to $\beta$-actin. (C) Quantification of p62 protein expression normalized to $\beta$-actin. Data are expressed as the mean \pm standard error of the mean. One-way analysis of variance was used to analyze the data. ${ }^{\#} \mathrm{P}<0.05$ vs. control; ${ }^{\&} \mathrm{P}<0.05$ vs. Group $\mathrm{A} ;{ }^{*} \mathrm{P}<0.05$ vs. Group B. Ctrl, control group treated with corn oil; group A, mice treated with a low dose of DMC; group $\mathrm{B}$, mice treated with a medium dose of $\mathrm{DMC}$; group $\mathrm{C}$, mice treated with a high dose of DMC; DMC, dimethyl carbonate. p62, a marker protein that indicates whether autophagosomes were successfully degraded (25). In group A, the expression of Beclin-1 was increased but p62 expression was similar to that of control group. By contrast, in groups B and C, Beclin-1 expression was decreased but p62 expression was increased compared with the control. This suggests that the accumulation of autophagosomes may increase the $\mathrm{Bax} / \mathrm{Bcl}-2$ ratio, thereby leading to the activation of caspase- 3 in the ovaries of mice in the high dose groups. It has been demonstrated that high concentrations of toxins may enable the maintenance of Beclin-1 expression despite the upregulation of LC-3II $(2,7)$, which is consistent with the results of the current study.

HIF-1 is a member of the basic-Helix-Loop-Helix-PAS family of transcription factors, which has been characterized as a transcriptional activator of several oxygen-sensitive genes, including transferrin, erythropoietin, hemeoxygenases and several glycolytic enzymes (26-29). The protein products of these genes serve vital roles in developmental and physiological processes, including iron transport, angiogenesis, erythropoiesis, glycolysis and cell proliferation/survival (30-34). It has been demonstrated that the expression of HIF-1 $\alpha$, a subunit of HIF-1, is induced by a decrease in $\mathrm{O}_{2}$ concentration, an increase in ROS and the stimulation of follicle-stimulating hormone in the mammalian ovary $(9,31,35,36)$. Under conditions of stress, the upregulation of HIF-1 $\alpha$ allows cells to successfully adapt to, or overcome their oxygen and nutrient-deprived state and survive in otherwise hostile environments (37). Studies have indicated that HIF-1 $\alpha$ may maintain cell survival by activating the downstream protein BNIP3 and subsequently inducing autophagy $(38,39)$. Exposure to toxins may significantly aggravate the cellular microenvironment by increasing ROS levels $(40,41)$, which may activate HIF-1 $\alpha$ expression. To determine whether the HIF-1 $\alpha /$ BNIP3 signaling pathway was involved in the induction of autophagy following exposure to DMC, the expression of HIF-1 $\alpha$ and BNIP3 were measured. The results indicated that levels of HIF-1 $\alpha$ and BNIP3 were increased in group A compared with the control group, and subsequently downregulated in groups B and $\mathrm{C}$ following exposure to increased doses of DMC, which is consistent with the tendency of Beclin-1. The activation of HIF-1 $\alpha /$ BNIP3 signaling may contribute to the induction of a self-protective response by autophagy, whereas high doses of DMC may disrupt the cytoprotective mechanism of the ovaries by upregulating the expression of p62, Bax and cleaved caspase-3, which are signals of aberrant autophagosome accumulation $(42,43)$. Thus, the role served by autophagy in the ovaries is dependent on the dose of DMC. Chen et al (44) demonstrated that autophagy induced by hypoxia served dual roles and was regulated by different signaling pathways depending on whether the duration of hypoxia was short or prolonged. The present study did not investigate the regulatory mechanisms underlying the downregulation of HIF-1 $\alpha / \mathrm{BNIP} 3$ signaling and the upregulation of LC-3II in the groups exposed to high doses of DMC; therefore further investigations are required.

In conclusion, to the best of our knowledge, the present study was the first to evaluate the toxicity of DMC exposure on ovarian function and the involvement of autophagy activation in ovarian injuries. The results demonstrated that DMC exposure 


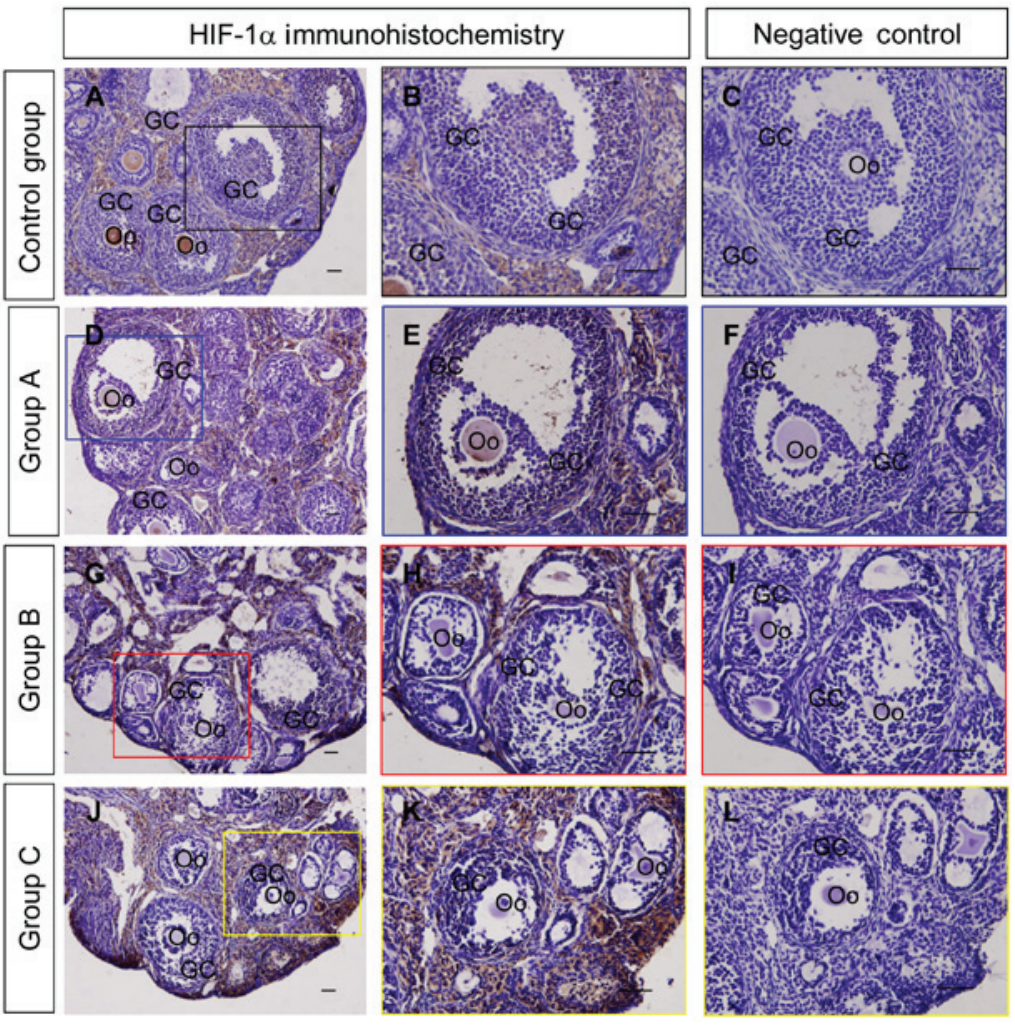

Figure 7. Immunohistochemical examination of HIF-1 $\alpha$ in the ovaries from each group. HIF-1 $\alpha$ immunohistochemical signals are brown and the hematoxylin counterstaining background are blue. Negative controls remained unstained as they lacked primary antibody. (A and B) HIF-1 $\alpha$ immunohistochemical staining of ovaries from the control group. (C) Negative control of the control group. (D and E) HIF-1 $\alpha$ immunohistochemical staining in ovaries from group A. (F) Negative control of group A. (G and H) HIF-1 $\alpha$ immunohistochemistry in ovaries from group B. (I) Negative control of group B. (J and K) HIF-1 $\alpha$ immunohistochemical staining in ovaries from group C. (L) Negative control of group C. Scale bar $=100 \mu \mathrm{m}$. Coloured boxes on the left side panels indicate the area exhibited on the middle and right side panels. GC, granulosa cell, Oo; oocyte; control group, mice treated with corn oil; group A, mice treated with a low dose of DMC; group B, mice treated with a medium dose of DMC; group C, mice treated with a high dose of DMC; DMC, dimethyl carbonate.
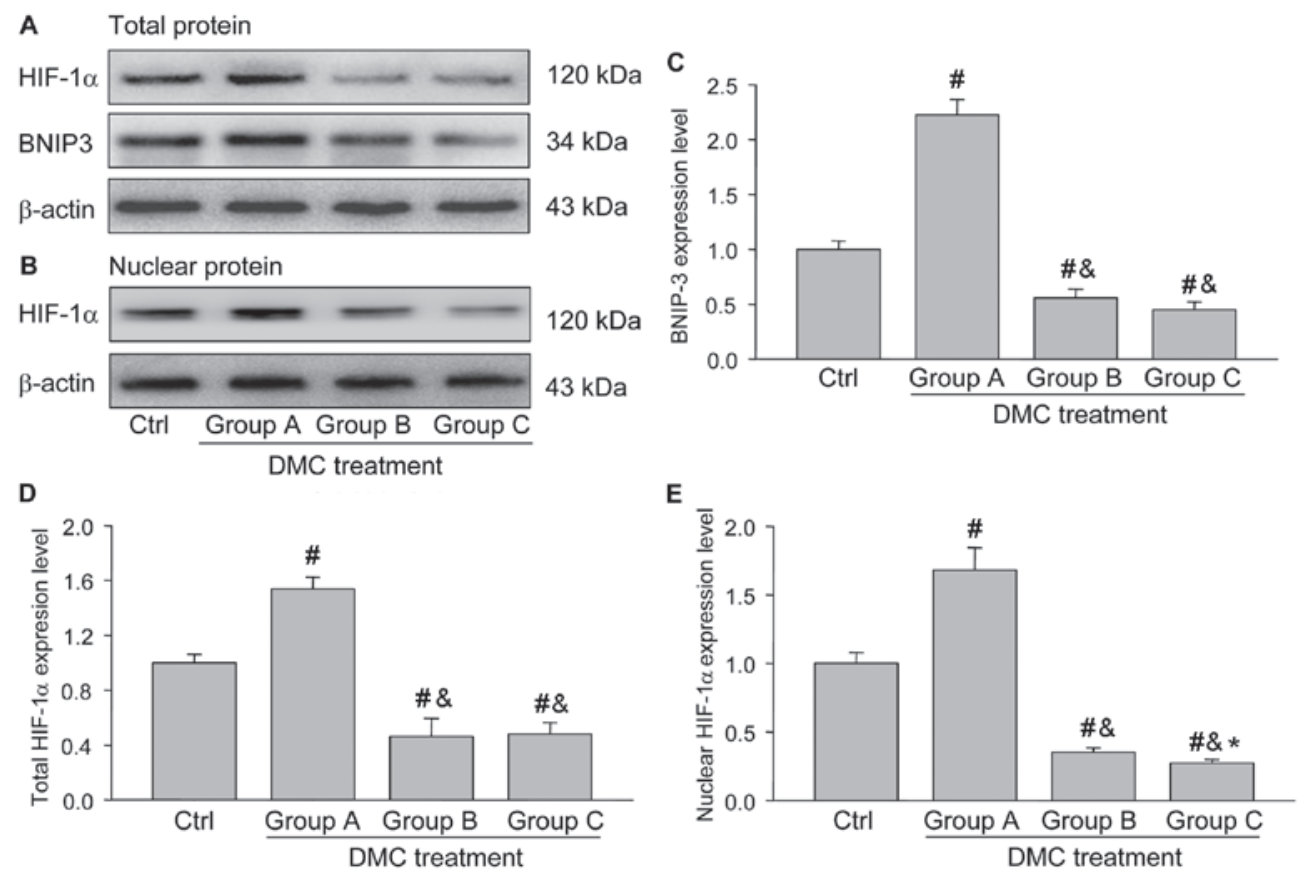

Figure 8. Western blotting analysis of HIF-1 $\alpha$ and BNIP3 expression in the ovaries from each group. (A) Western blotting indicating the expression of HIF-1 $\alpha$ and BNIP3 in the different groups. (B) Western blotting indicating the expression of HIF-1 $\alpha$ nuclear protein in the different groups. (C) Quantification of BNIP3 expression normalized to $\beta$-actin. (D) Quantification of total HIF-1 $\alpha$ expression normalized to $\beta$-actin. (E) Quantification of HIF-1 $\alpha$ nuclear expression normalized to $\beta$-actin. Data are expressed as the mean \pm standard error of the mean. One-way analysis of variance was used to analyze the data. ${ }^{*} \mathrm{P}<0.05$ vs. control; ${ }^{\circledR} \mathrm{P}<0.05$ vs. Group A; ${ }^{*} \mathrm{P}<0.05$ vs. Group B. Ctrl, control group treated with corn oil; group A, mice treated with a low dose of DMC; group B, mice treated with a medium dose of DMC; group C, mice treated with a high dose of DMC; DMC, dimethyl carbonate; HIF-1 $\alpha$, hypoxia inducible factor $1 \alpha$ subunit; BNIP3, BCL2 interacting protein 3. 
induces the activation of autophagy in the ovaries, which may protect follicular development following exposure to low doses of DMC via activation of HIF-1 $\alpha$ /BNIP3 signaling pathway. DMC exposure also induced follicular atresia in the groups exposed to medium and high doses of DMC by activating the apoptotic signaling pathway. This may be an important mechanism regulating follicular development and ovarian function in the mammalian ovaries.

\section{Acknowledgements}

The present study was supported by the National Natural Science Foundation of China (grant no. 31271255; Beijing, China), Fujian Provincial Natural Science Foundation (grant nos. 2016J01145 and 2017J01626; Fujian, China) and Fujian Province Science and Technology Project of The Education Department (grant nos. JAT160118 and JZ160426, Fujian, China).

\section{References}

1. Zhang G, Liu K, Ling X, Wang Z, Zou P, Wang X, Gao J, Yin L, Zhang X, Liu J, et al: DBP-induced endoplasmic reticulum stress in male germ cells causes autophagy, which has a cytoprotective role against apoptosis in vitro and in vivo. Toxicol Lett 245 86-98, 2016

2. Zhang S, Niu Q, Gao H, Ma R, Lei R, Zhang C, Xia T, Li P, Xu C, Wang $C$, et al: Excessive apoptosis and defective autophagy contribute to developmental testicular toxicity induced by fluoride. Environ Pollut 212: 97-104, 2016.

3. Lall R, Ganapathy S, Yang M, Xiao S, Xu T, Su H, Shadfan M, Asara JM, Ha CS, Ben-Sahra I, et al: Low-dose radiation exposure induces a HIF-1-mediated adaptive and protective metabolic response. Cell Death Differ 21: 836-844, 2014.

4. Bellot G, Garcia-Medina R, Gounon P, Chiche J, Roux D, Pouysségur J and Mazure NM: Hypoxia-induced autophagy is mediated through hypoxia-inducible factor induction of BNIP3 and BNIP3L via their BH3 domains. Mol Cell Biol 29: 2570-2581, 2009.

5. Bevan C and Beyer B: Developmental toxicity evaluation of dimethylcarbonate by inhalation in CD-1 mice. Int Toxicol 7, 72: 1995.

6. Anderson SE, Franko J, Anderson KL, Munson AE, Lukomska E and Meade BJ: Immunotoxicity and allergic potential induced by topical application of dimethyl carbonate (DMC) in a murine model. J Immunotoxicol 10: 59-66, 2013.

7. Quan C, Wang C, Duan P, Huang W, Chen W, Tang S and Yang K: Bisphenol a induces autophagy and apoptosis concurrently involving the Akt/mTOR pathway in testes of pubertal SD rats. Environ Toxicol 32: 1977-1989, 2017.

8. Liu ML, Wang JL, Wei J, Xu LL, Yu M, Liu XM, Ruan WL and Chen JX: Tri-ortho-cresyl phosphate induces autophagy of rat spermatogonial stem cells. Reproduction 149 $163-170,2015$

9. Duan P, Hu C, Quan C, Yu T, Zhou W, Yuan M, Shi Y and Yang K: 4-Nonylphenol induces apoptosis, autophagy and necrosis in Sertoli cells: Involvement of ROS-mediated AMPK/AKT-mTOR and JNK pathways. Toxicology 341-343: 28-40, 2016.

10. Li Y, Wang Y, Kim E, Beemiller P, Wang CY, Swanson J, You M and Guan KL: Bnip3 mediates the hypoxia-induced inhibition on mammalian target of rapamycin by interacting with Rheb. J Biol Chem 282: 35803-35813, 2007

11. Zhang M, Jiang M, Bi Y, Zhu H, Zhou Z and Sha J: Autophagy and apoptosis act as partners to induce germ cell death after heat stress in mice. PLoS One 7: e41412, 2012.

12. Wang SH, Shih YL, Ko WC, Wei YH and Shih CM: Cadmium-induced autophagy and apoptosis are mediated by a calcium signaling pathway. Cell Mol Life Sci 65: 3640-3652, 2008.

13. Kabeya Y, Mizushima N, Ueno T, Yamamoto A, Kirisako T, Noda T, Kominami E, Ohsumi Y and Yoshimori T: LC3, a mammalian homologue of yeast Apg8p, is localized in autophagosome membranes after processing. EMBO J 19: 5720-5728, 2000 .
14. Xu LL, Liu ML, Wang JL, Yu M and Chen JX: Saligenin cyclic-o-tolyl phosphate (SCOTP) induces autophagy of rat spermatogonial stem cells. Reprod Toxicol 60: 62-68, 2016.

15. Zhang G, Liu K, Ling X, Wang Z, Zou P, Wang X, Gao J, Yin L, Zhang X, Liu J, et al: DBP-induced endoplasmic reticulum stress in male germ cells causes autophagy, which has a cytoprotective role against apoptosis in vitro and in vivo. Toxicol Lett 245: 86-98, 2016.

16. Chen Y, Zhou Y, Wang X, Qian W and Han X: Microcystin-LR induces autophagy and apoptosis in rat Sertoli cells in vitro. Toxicon 76: 84-93, 2013.

17. Gannon AM, Stämpfli MR and Foster WG: Cigarette smoke exposure elicits increased autophagy and dysregulation of mitochondrial dynamics in murine granulosa cells. Biol Reprod 88: 63, 2013.

18. Obert LA, Sobocinski GP, Bobrowski WF, Metz AL, Rolsma MD, Altrogge DM and Dunstan RW: An immunohistochemical approach to differentiate hepatic lipidosis from hepatic phospholipidosis in rats. Toxicol Pathol 35: 728-734, 2007.

19. Nahdi A, Hammami I, Kouidhi W, Chargui A, Ben Ammar A, Hamdaoui MH, El May A and El May M: Protective effects of crude garlic by reducing iron-mediated oxidative stress, proliferation and autophagy in rats. J Mol Histol 41: 233-245, 2010.

20. Yin XM, Oltvai ZN and Korsmeyer SJ: BH1 and BH2 domains of Bcl-2 are required for inhibition of apoptosis and heterodimerization with Bax. Nature 369: 321-323, 1994.

21. Booth LA, Tavallai S, Hamed HA, Cruickshanks N and Dent P: The role of cell signalling in the crosstalk between autophagy and apoptosis. Cell Signal 26: 549-555, 2014.

22. Canipari R: Oocyte-granulosa cell interactions. Hum Reprod Update 6: 279-289, 2000.

23. Wang C and Klionsky DJ: The molecular mechanism of autophagy. Mol Med 9: 65-76, 2003.

24. Nazarko VY and Zhong Q: ULK1 targets Beclin-1 in autophagy. Nat Cell Biol 15: 727-728, 2013.

25. Pankiv S, Clausen TH, Lamark T, Brech A, Bruun JA, Outzen H, Øvervatn A, Bjørkøy G and Johansen T: p62/SQSTM1 binds directly to Atg8/LC3 to facilitate degradation of ubiquitinated protein aggregates by autophagy. J Biol Chem 282: 24131-24145, 2007.

26. Wang GL, Jiang BH, Rue EA and Semenza GL: Hypoxia-inducible factor 1 is a basic-helix-loop-helix-PAS heterodimer regulated by cellular O2 tension. Proc Natl Acad Sci USA 92: 5510-5514, 1995.

27. Wang Z, Tang L, Zhu Q, Yi F, Zhang F, Li PL and Li N: Hypoxia-inducible factor- $1 \alpha$ contributes to the profibrotic action of angiotensin II in renal medullary interstitial cells. Kidney Int 79: 300-310, 2011.

28. Wang Z, Zhu Q, Li PL, Dhaduk R, Zhang F, Gehr TW and Li N: Silencing of hypoxia-inducible factor- $1 \alpha$ gene attenuates chronic ischemic renal injury in two-kidney, one-clip rats. Am J Physiol Renal Physiol 306: F1236-F1242, 2014.

29. Wang Z, Zhu Q, Xia M, Li PL, Hinton SJ and Li N: Hypoxia-inducible factor prolyl-hydroxylase 2 senses high-salt intake to increase hypoxia inducible factor 1alpha levels in the renal medulla. Hypertension 55: 1129-1136, 2010.

30. Yaba A, Bianchi V, Borini A and Johnson J: A putative mitotic checkpoint dependent on mTOR function controls cell proliferation and survival in ovarian granulosa cells. Reprod Sci 15: 128-138, 2008.

31. Zhong H, Chiles K, Feldser D, Laughner E, Hanrahan C, Georgescu MM, Simons JW and Semenza GL: Modulation of hypoxia-inducible factor 1alpha expression by the epidermal growth factor/phosphatidylinositol 3-kinase/PTEN/AKT/FRAP pathway in human prostate cancer cells: Implications for tumor angiogenesis and therapeutics. Cancer Res 60: 1541-1545, 2000.

32. Wulff C, Dickson SE, Duncan WC and Fraser HM: Angiogenesis in the human corpus luteum: Simulated early pregnancy by HCG treatment is associated with both angiogenesis and vessel stabilization. Hum Reprod 16: 2515-2524, 2001.

33. Nishimura R and Okuda K: Hypoxia is important for establishing vascularization during corpus luteum formation in cattle. J Reprod Dev 56: 110-116, 2010.

34. Miyazawa M, Yasuda M, Fujita M, Hirabayashi K, Hirasawa T, Kajiwara H, Muramatsu T, Miyazaki S, Harasawa M, Matsui N, et al: Granulosa cell tumor with activated mTOR-HIF-1alpha-VEGF pathway. J Obstet Gynaecol Res 36: 448-453, 2010. 
35. Jewell UR, Kvietikova I, Scheid A, Bauer C, Wenger RH and Gassmann M: Induction of HIF-1alpha in response to hypoxia is instantaneous. FASEB J 15: 1312-1314, 2001.

36. Zhang Z, Wang Q, Ma J, Yi X, Zhu Y, Xi X, Feng Y and Jin Z: Reactive oxygen species regulate FSH-induced expression of vascular endothelial growth factor via Nrf2 and HIF1 $\alpha$ signaling in human epithelial ovarian cancer. Oncol Rep 29: 1429-1434, 2013.

37. Ietta F, Wu Y, Winter J, Xu J, Wang J, Post M and Caniggia I: Dynamic HIF1A regulation during human placental development. Biol Reprod 75: 112-121, 2006.

38. Chen J, Bai M, Ning C, Xie B, Zhang J, Liao H, Xiong J, Tao X, Yan D, Xi X, et al: Gankyrin facilitates follicle-stimulating hormone-driven ovarian cancer cell proliferation through the PI3K/AKT/HIF-1 $\alpha /$ cyclin D1 pathway. Oncogene 35: 2506-2517, 2016.

39. Bellot G, Garcia-Medina R, Gounon P, Chiche J, Roux D, Pouysségur J and Mazure NM: Hypoxia-induced autophagy is mediated through hypoxia-inducible factor induction of BNIP3 and BNIP3L via their BH3 domains. Mol Cell Biol 29: 2570-2581, 2009.
40. Zhao Y, Chen G, Zhang W, Xu N, Zhu JY, Jia J, Sun ZJ, Wang YN and Zhao YF: Autophagy regulates hypoxia-induced osteoclastogenesis through the HIF-1 $\alpha /$ BNIP3 signaling pathway. J Cell Physiol 227: 639-648, 2012.

41. Liu X, Xu L, Shen J, Wang J, Ruan W, Yu M and Chen J: Involvement of oxidative stress in tri-ortho-cresyl phosphate-induced autophagy of mouse Leydig TM3 cells in vitro. Reprod Biol Endocrin 14: 30, 2016.

42. Sun L,LiT, Wei Q, Zhang Y,Jia X, Wan Zand Han L: Upregulation of BNIP3 mediated by ERK/HIF-1 $\alpha$ pathway induces autophagy and contributes to anoikis resistance of hepatocellular carcinoma cells. Future Oncol 10: 1387-1398, 2014.

43. Mathew R, Karp CM, Beaudoin B, Vuong N, Chen G, Chen HY, Bray K, Reddy A, Bhanot G, Gelinas C, et al: Autophagy suppresses tumorigenesis through elimination of p62. Cell 137: 1062-1075, 2009

44. Chen G, Zhang W, Li YP, Ren JG, Xu N, Liu H, Wang FQ, Sun ZJ, Jia J and Zhao YF: Hypoxia-induced autophagy in endothelial cells: A double-edged sword in the progression of infantile haemangioma? Cardiovasc Res 98: 437-448, 2013. 